\title{
BMJ Open Protocol for the BRECAR study: a prospective cohort follow-up on the impact of breast reconstruction timing on health-related quality of life in women with breast cancer
}

\author{
Maria Herrera de la Muela, ${ }^{1,2}$ Enrique García López, ${ }^{1}$ Laura Frías Aldeguer, ${ }^{1}$ \\ Paloma Gómez-Campelo, ${ }^{2}$ On behalf of the BRECAR Study Group
}

To cite: Herrera de la Muela M, García López E, Frías Aldeguer L, et al. Protocol for the BRECAR study: a prospective cohort follow-up on the impact of breast reconstruction timing on health-related quality of life in women with breast cancer. BMJ Open 2017;7:e018108. doi:10.1136/ bmjopen-2017-018108

- Prepublication history for this paper is available online. To view these files, please visit the journal online (http://dx.doi. org/10.1136/bmjopen-2017018108).

Received 8 June 2017 Accepted 13 0ctober 2017

\section{(a) CrossMark}

${ }^{1}$ Breast Pathology Unit, Department of Gynecology, Hospital Universitario La Paz, Madrid, Spain

${ }^{2}$ Hospital La Paz Institute for Health Research (IdiPAZ), La Paz University Hospital, Madrid, Spain

Correspondence to Dr Paloma Gómez-Campelo; pgomezc@salud.madrid.org

\section{ABSTRACT}

Introduction The completion of postmastectomy breast reconstruction (BR) in women with breast cancer can last from months to years, and to our knowledge, there is a lack of studies that analyse how the different types and times of reconstruction impact on the patient's quality of life and psychosocial adjustment. The primary aim of the BREast Cancer Reconstruction (BRECAR Study) is twofold. First, to describe health-related quality of life (HRQLL), overall satisfaction with surgery and psychological impact (body image, self-esteem, depression and anxiety) on women who will have undergone a mastectomy with planned BR, considering the varied timing of $\mathrm{BR}$ procedures (immediate $\mathrm{BR}(\mathrm{BR})$, delayed BR (dBR) and two-stage BR (2sBR)). To measure the impact on surgical outcomes, we will obtain data prior to and after surgery (6-9 and at 18 months of follow-up). Second, to analyse sociodemographic, clinical and psychosocial factors associated with HRQoL, satisfaction with surgery and psychological impact.

Methods and analysis A prospective, observational, clinical cohort study of women diagnosed with breast cancer who have an indication for mastectomy treated at La Paz University Hospital (Madrid, Spain). Patients will be classified into one of three groups under conditions of routine clinical practice, based on the type of BR planned: the iBR group, the $\mathrm{dBR}$ group and the 2sBR group. Under typical clinical practice conditions, we will perform three visits: baseline visit (presurgery), V1 (6-9 months after diagnosis) and V2 (18 months after diagnosis). A sample size of 210 patients is estimated.

Ethics and dissemination The study protocol and informed consent form have been reviewed and approved by the Institutional Review Board of La Paz Hospital (no. PI -2036). Dissemination of results will be via journal articles and conference presentations.

\section{INTRODUCTION}

Breast cancer is the most frequent malignant tumour among women. In 2012, an estimated 1671000 cases were diagnosed and approximately 522000 women worldwide died of breast cancer. $^{1}$
Strengths and limitations of this study

- A gap on prospective studies focusing on how the type and timing of breast reconstruction affect the patients.

- Cohort study involving data about quality of life, self-esteem, body image and satisfaction with the surgery.

- Classification of the patients in the groups according to breast reconstruction timing will be performed under routine medical conditions.

- A possible limitation could be the hospital-based sample limiting the generalisability of findings.

- Will produce valuable data regarding the impact of different types and times of reconstruction.

In Spain, the incidence is 25215 cases per year that represents nearly $30 \%$ of all tumours in women, with an estimated 5-year prevalence of 104210 cases. Breast cancer is also the leading cause of cancer-related deaths, with a rate of 17.6 deaths per 100000 women. ${ }^{2}$ However, the current long-term prognosis for women with breast cancer has improved significantly in recent decades, particularly the survival of women in operable stages $(0$ -III), which fluctuates around $70 \%{ }^{2}$

The surgical treatment of breast cancer has evolved over the past century in an attempt to improve cosmetic outcomes and reduce surgical morbidity while still ensuring an oncologically sound surgical procedure. ${ }^{3}$ According to data from the Spanish Society of Reconstructive, Aesthetic and Plastic Surgery, ${ }^{4}$ approximately $60 \%$ of patients with breast cancer will require a mastectomy, and approximately $70 \%$ of these patients will have breast reconstruction (BR), $10 \%$ will undergo immediate BR surgery and 20\%-25\% will undergo delayed BR. 
From a medical point of view, BR is offered to all patients under the age of 70 years with an indication for mastectomy, ${ }^{567}$ and the patient's age is not an absolute limitation for surgery. There are some contraindications and limitations, however, such as advanced stages of disease, obesity, hypertension, diabetes, smoking and the need for adjuvant radiotherapy.

As for the type of BR, three types are included: autologous (with patient tissue, using flaps from the abdomen, thigh, gluteal region, dorsal region and so on), alloplastic (using implants, both expanders and prostheses) or mixed (combining autologous tissue with prosthetic implants). ${ }^{8910}$ The choice of which postmastectomy BR used will depend on the individual patient characteristics and the experience of the surgical team.

Regarding the timing of BR, it can be performed at the same time as the mastectomy (immediate BR (iBR)), after the culmination of the adjuvant treatment with chemotherapy and/or radiotherapy (delayed BR $(\mathrm{dBR})$ ) or in two stages (two-stage BR (2sBR)); in the first stage, an expander implant is placed during mastectomy, then, approximately 6 months after the first surgery or after completion of adjuvant treatment, a second surgery is performed, replacing the expander with a prosthesis or autologous tissue. In all cases, the reconstruction of the areola-nipple complex is proposed approximately 6-12 months after the finalisation of adjuvant treatments. ${ }^{11} 12$ Therefore, depending on the timing of $\mathrm{BR}$, the surgical treatment can be prolonged from months to years until the process is finished.

Historically, dBR was performed 2 years after mastectomy, which caused a considerable alteration in the patient's health-related quality of life (HRQoL), delaying the recovery of a normal life. ${ }^{13}$ Today, the intent of postmastectomy BR is applied in most cases, but the main limiting factor considered when choosing the type of BR is the indication for adjuvant radiotherapy that in many patients is not prescribed until the finding of adverse pathology. ${ }^{14} 15$

Radiotherapy above autologous tissues can lead to an increase in some complications (eg, necrosis, fibrosis, infections and loss of volume) and can alter the final outcome; however, whether reconstruction is performed immediately or deferred appears to make no difference. $^{1617181920}$

Although some small series show conflicting results, ${ }^{21}$ most studies indicate that radiotherapy treatment of patients with prosthetic implants increases the risk of complications (eg, capsular contracture, implant rejection and infections). ${ }^{22} 2315$ Thus, surgeons frequently will not use permanent prosthetic implants if there is a risk of the need for radiotherapy, opting instead for a 2sBR or dBR with autologous tissue. ${ }^{24}$ Other authors, however, find this controversial and argue that there are no advantages to deferred reconstruction when radiotherapy needs to be performed..$^{26}$ Therefore, in terms of BR, and with equal indication and type of patient and adjuvant treatment, there is not a single option, and variability is the norm.
The consensus of the Spanish Society of Senology and Mammary Pathology regarding mammary reconstruction recommends using autologous or mixed reconstruction when radiotherapy is needed, avoiding the use of expanders or prosthetics. ${ }^{11}$ However, some groups have shown excellent results using implants with radiotherapy or dBR. Thus, factors related to the patient's own preference and the surgical complexity she is willing to assume should be considered. ${ }^{27}$ The preference and experience of the reconstructive team should also influence the decision.

From a psychological point of view, a set of psychosexual changes have been documented following mastectomy, including negative body image, loss of femininity and attractiveness, depression and anxiety. In addition, some symptoms such as sexual dysfunction, vaginal dryness, decreased sexual desire and/or sexual pleasure have been reported. ${ }^{28} 293031$

Treatment strategies in women with breast cancer include BR, which aims to enhance recovery or maintenance of an acceptable level of HRQoL, including phys ical, psychological, social and sexual well-being. We cannot forget that many other dimensions such as body image, self-esteem, patient satisfaction (including satisfaction with aesthetic results, information received and medical equipment) are included in this concept. ${ }^{32} 3334$

Specialised scientific literature demonstrates, however, that BR is not always the best solution for quality of life improvement, because it has been considered the gold standard to evaluate surgery impact, surgery satisfaction and psychosocial adjustment, not quality of life. Therefore, there is some controversy regarding the type and timing of the most suitable reconstruction for each patient. ${ }^{34} 3536$

Most research has examined the psychosocial outcomes of mastectomy compared with conservative breast surgery, showing clear psychosocial benefits and a better quality of life in the latter. ${ }^{32}{ }^{37}$ In addition, in women who have undergone mastectomy, those with BR experience less anxiety and depression, and better body image, self-es teem and quality of life than those without BR..$^{38}$

There is a gap, however, in the specialised literature regarding studies focusing on how the various types and timing of BR affect the patient's quality of life and psychosocial adjustment. The few studies in this field have been developed at an international level and, as far as we know, there are no published studies on Spain in this regard.

There has recently been an increased emphasis on patient-reported outcomes with respect to surgical BR techniques related to satisfaction with the breast and outcomes, psychological well-being, physical well-being and sexual well-being. The scarce data available show that women with iBR have less distress, better self-esteem, better psychosocial well-being and greater satisfaction than those with dBR. ${ }^{38} 394041$ Therefore, iBR appears to offer psychosocial advantages in quality of life and in the patient's general well-being compared with other BRs. Nevertheless, it should be remembered that not 
all patients are candidates for this type of intervention. Prospective studies, with follow-up times from surgery between 6 months and 1 year, ${ }^{3941424344}$ are scarce. Teo et $a l,{ }^{41}$ in a study of 216 patients followed from the preoperative period to the completion of reconstructive treatment, concluded that both the time and the phase of reconstruction are two fundamental factors that must be taken into consideration when discussing psychosocial impact and its effect on the patient's well-being and quality of life. In addition, they indicate that those patients who undergo dBR will present poorer adjustment than those with immediate reconstruction, even during the preoperational phase of the process.

In summary, understanding the outcomes regarding quality of life and patients' psychosocial adjustment associated with BR is essential to consider, along with clinical factors, the type and timing of BR for each woman, thus facilitating the decision-making process as much as possible. In this way, it will be possible to design and implement new therapeutic strategies for the large group of breast cancer survivors treated with radical surgery, taking into account the influence of the type and timing of the reconstruction of the breast.

To our knowledge, this is the first cohort study conducted in Spain to follow patients with breast cancer who have indication for mastectomy and BR that will analyse data regarding psychosocial functioning on preoperative and postoperative mastectomy, with presurgical evaluation and short-term and medium-term follow-ups (18 months). We focus on the development of a predictive model as a tool to help the clinician and the patient to decide what type and timing of BR are the most appropriate in their case, personalising the procedure intervention and adjusting to the patient.

\section{OBJECTIVES}

The primary aim of the BRECAR study is twofold. First, to describe the HRQoL, the overall satisfaction with surgery and the psychological impact (body image, self-esteem, depression and anxiety) in women who will have undergone a mastectomy with planned $\mathrm{BR}$, considering the varied timing of $\mathrm{BR}$ procedures (iBR, $\mathrm{dBR}$ or $2 \mathrm{sBR}$ ). To measure the impact on surgical outcomes, we will obtain data prior to and after breast cancer surgery (6-9 and 18 months follow-up). Second, to analyse sociodemographic, clinical and psychosocial factors associated with HRQoL, satisfaction with surgery and psychological impact.

Secondary objectives:

1. To describe the rate of BR (autologous, alloplastic or mixed).

2. To describe the impact on clinical outcomes (postop erative complications and secondary effects of adjuvant therapy) and psychological impact (body image, self-esteem, depression and anxiety), according to the type of BR (autologous, alloplastic or mixed).
3. To analyse sociodemographic, clinical and psychosocial factors related to abandonment of planned BR surgery.

\section{METHODS}

\section{Study design}

This study is a 3-year, observational, prospective cohort study. We have applied Strengthening the Reporting of Observational Studies in Epidemiology (STROBE) guidelines to this protocol (http://www.strobestatement.org). The study is being performed from 1 January 2017 to 31 June 2021, and recruitment of the sample is being conducted between January and December of 2019.

\section{Setting}

The study will be performed at the Breast Pathology Unit of La Paz University Hospital's Department of Gynecology (Madrid, Spain).

\section{Participants}

Participants will be eligible for the study if they meet the following inclusion criteria:

- Women over the age of 18.

- Outpatients with breast cancer who will undergoing a mastectomy with planned BR.

- A relatively recent diagnosis of unilateral breast cancer (maximum time since diagnosis 3 months).

- Agreement to participate in the study and to provide written informed consent.

Participants will be excluded if they meet any of the following exclusion criteria:

- Absolute contraindications to BR, such as presence of severe psychopathology or dysmorphic disorder and /or severe systemic disease with contraindications for anaesthesia (according to clinical criteria).

- Stage IV cancer.

- Previous diagnosis of cancer or concurrent diagnosis of another cancer.

- Mastectomy after breast cancer recurrence.

- Patients with severe chronic diseases or significant physical or psychological disabilities that might invalidate informed consent or interview outcomes (according to clinical judgement).

- Participants who cannot understand Spanish.

\section{Procedure}

During routine hospital check-ups, potential participants will be approached by gynaecologist. Those who meet inclusion criteria, after an explanation of the study, will be invited to take part. Before evaluation, the gynaecologist will present the study, answer the participant's questions and present the consent form for signature. After obtaining the informed consent, the women who have agreed to take part in the study will be escorted to a clinical research room for completion of the paper case report forms. The estimated average time spent on assessment will be $30-45 \mathrm{~min}$. 


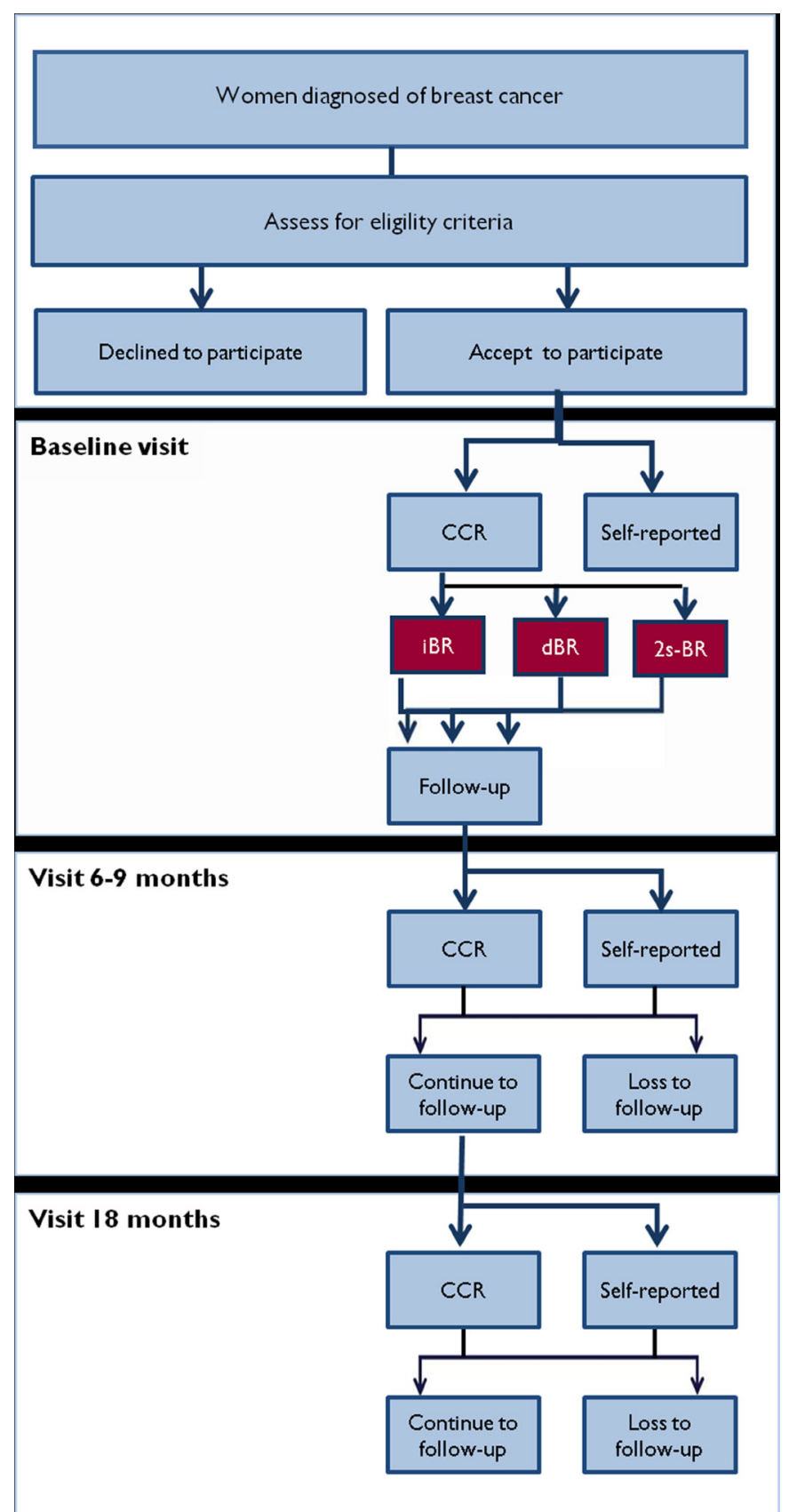

Figure 1 BRECAR study flow chart. 2sBR, two-stage BR; BR, breast reconstruction; BRECAR, BREast Cancer Reconstruction; CCR, computerised clinical records; dBR, delayed BR; iBR, immediate BR.

Evaluation and follow-up visits by clinicians are to be scheduled as follows (figure 1):

- Baseline visit: after diagnosis and previous to mastectomy (presurgery visit).

- Visit 1: 6-9 months after diagnosis.

- Visit 2: 18 months after diagnosis.

Patients with breast cancer who will undergo a mastectomy with a planned BR will be identified prospectively at attending follow-up visits by clinicians. The BR procedure will be offered to women under routine medical conditions, following published consensus data. ${ }^{11}$ We offer iBR to be considered by the patient except when significant comorbidity of the patient or adjuvant therapy precludes this option. Finally, patients will be identified and classified into three groups based on the BR timing:

The iBR group: insertion of permanent implant or autologous tissue at initial surgery.

The dBR group: reconstruction (with implant or autologous tissue) is done after mastectomy during a separate procedure, once women have completed any additional treatments.

The 2sBR group: insertion of a temporary expander with a plan to perform a definitive reconstruction (with autologous tissue or permanent implant) after completion of the adjuvant treatment.

All BRs will be performed by consultant plastic surgeons with special training in BR procedures.

\section{Variables}

The primary outcome measures will be HRQoL, psychological adjustment (body image dissatisfaction, self-esteem and depression) and satisfaction and well-being in $\mathrm{BR}$, as summarised in table 1 .

Health-related quality of life

HRQoL will be evaluated with the Spanish versions of the European Organization for Research and Treatment of Cancer (EORTC) Quality of Life Questionnaire version 3.0 (QLQ-30) ${ }^{45} 46$ and its supplementary Breast Cancer Module (QLQ-BR23) 4748

The QLQ-C30 is a well-known instrument for measuring quality of life in patients with cancer. It is a 30-item questionnaire with a four-point scale, from 'not at all' to 'very much' for items 1 to 28, and a seven-point scale for items 29 and 30. Each patient's scores are transformed into a 0-100 scale, in which 0 denotes the poorest and 100 denotes the best on functioning scales. In contrast, the reverse scoring system was applied for symptoms in which the zero point denotes the best and 100 denotes the poorest on symptom scales. The scoring approach was identical for the QLQ-BR23.

The QLQ-C30 dimensions include the following: physical functioning, role functioning, cognitive functioning, emotional functioning, social functioning, the global level of HRQoL and the symptoms scale (eg, fatigue and pain).

The QLQ-BR23 includes 23 items assessing four functional scales (body image, sexual functioning, sexual enjoyment and future perspectives) and four symptom scales (systemic therapy adverse effects, breast symptoms, arm symptoms and hair loss); as for the QLQ-C30, one score is generated per dimension on a $0-100$ scale in which a high score represents a high level of functioning and a high symptomatic level. ${ }^{46} 48$

\section{Body image dissatisfaction}

Body image will be evaluated using the Spanish version of the Body Image Scale (BIS) ${ }^{49}$, which is a 10 -item cancer-specific scale evaluating the impact of a surgical procedure on the patient's body image. The scale consists 


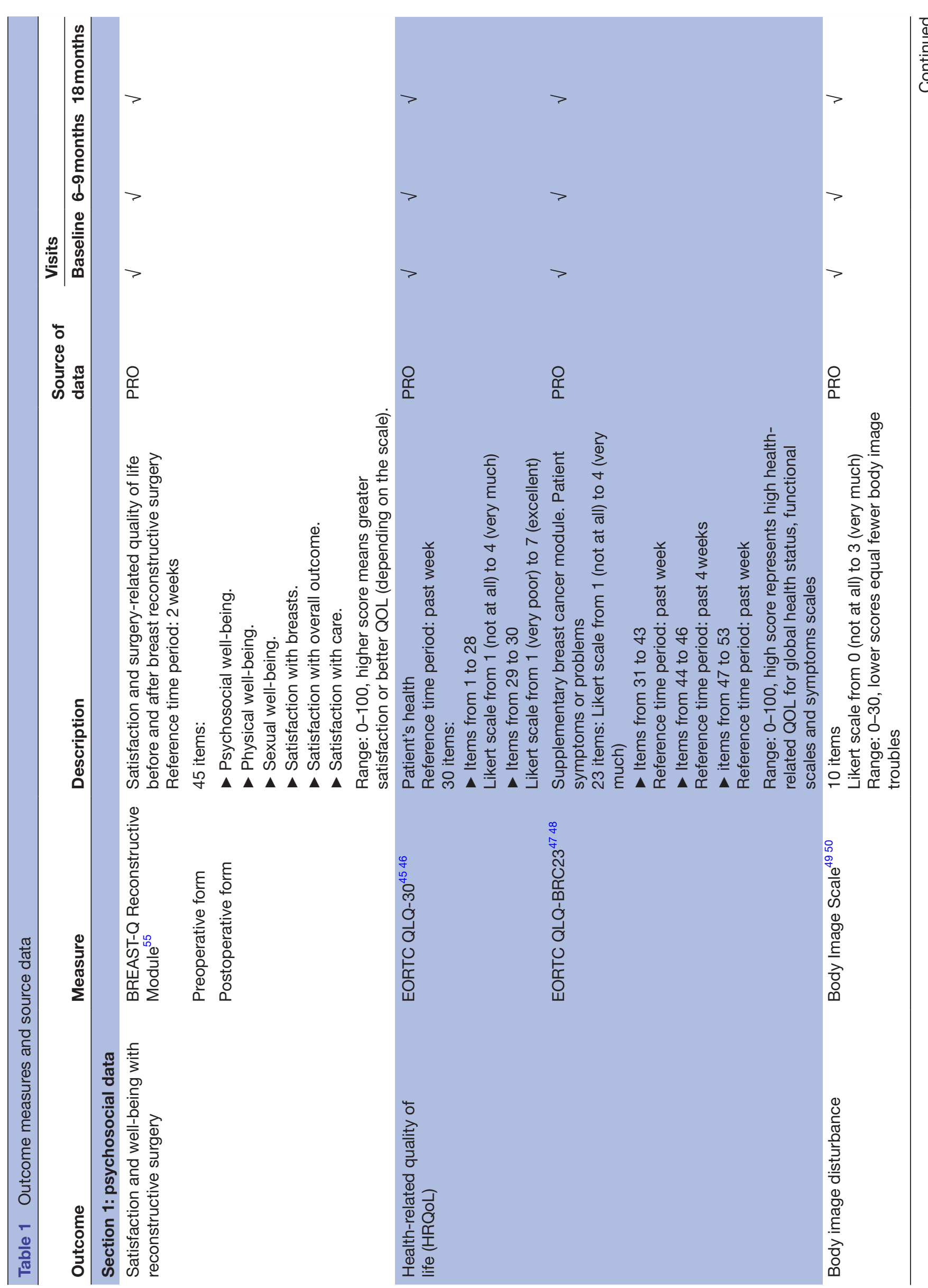




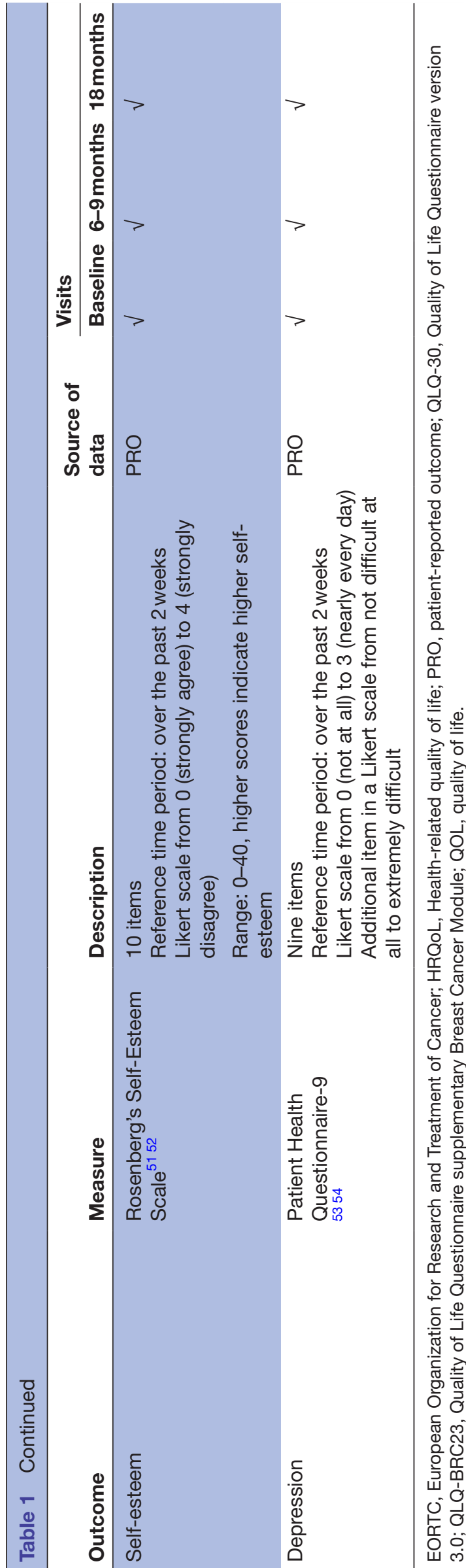

of items evaluating during the past week femininity, self-consciousness, physical and sexual attractiveness, satisfaction with body and scars. Each item is scored on a 4-point Likert scale from 0 (not at all) to 3 (very much). The sum of the BIS items provides a total score (range 0-30, lower scores represent fewer body image disturbances).

\section{Self-esteem}

The Spanish version of the Rosenberg Self-esteem Scale will be used to evaluate individual self-esteem. ${ }^{51}{ }^{52}$ Rosenberg's Self-esteem Scale comprises 10 items with a four-point intensity scale (strongly agree to strongly disagree), with a total score ranging from 0 to 40 points. Higher scores indicate better self-esteem.

\section{Depression}

The Patient Health Questionnaire (PHQ-9), in its Spanish version ${ }^{53}$, was used to evaluate the presence of depressive symptoms during the prior 2 weeks. The PHQ-9 is nine items, based on each of the Diagnostic and Statistical Manual IV diagnostic criteria for major depressive episode, which can be scored from 0 (not at all) to 3 (nearly every day); as a severity measure, scores can range from 0 (absence of depressive symptoms) to 27 (severe depressive symptoms). As a diagnostic measure, major depression is diagnosed if five or more of the nine depressive symptom criteria have been present at least 'more than half the days' (a score of 2) in the past 2 weeks, and one of the symptoms is depressed mood or anhedonia. The PHQ-9 is well validated and widely used as a brief diagnostic and severity measure of depression.

\section{Satisfaction with BR}

The BREAST- $\mathrm{Q}^{55}$ is the only questionnaire that has been specifically designed to assess patient-reported outcomes in plastic and reconstructive breast surgery. Four of the six subscales measure well-being and satisfaction before and after reconstruction: satisfaction with breasts, psychosocial well-being, sexual well-being, physical well-being with respect to chest and physical well-being with respect to the abdomen donor site. Two additional subscales measure post-BR outcomes related to satisfaction with outcome and satisfaction with information. All scales are scored from 0 to 100 , with higher scores indicating greater satisfaction or function.

To our knowledge, the BREAST-Q had not been translated and adapted to Spanish in Spain; thus, we proceeded to perform a full linguistic validation process according to the standard recognised methodology of translation of the measure according to the linguistic validation guidelines of the Mapi Research Trust. ${ }^{56}$ Considering the aim of the BRECAR Study, we developed a Spanish language version of the BREAST-Q Reconstruction Module, Preoperative (1.0) and Postoperative (2.0) forms. The validation process of the BREAST-Q was performed between September and November 2016 
The linguistic validation consisted of three steps. The initial stage, forward translation, included two Englishspeaking natives translating the source document, each of the translators producing an independent forward translation of the original items and response choices. Both translations were reviewed by an expert and were merged into a reconciliation version. The reconciliation Spanish version was then back-translated into English by a native English-speaking, bilingual Spanish translator. The project manager and backward translator then compared the backward version and the original English version to confirm whether the meanings and concepts were equivalent. Finally, after the backward version had been approved by the author of the original BREAST-Q patient testing was initiated to examine the content validity, acceptability and patient burden. A pilot test was conducted on a reduced sample of patients ( $n=10$; excluded from study) to assess comprehension of the translation, together with a brief questionnaire to ascertain the difficulties encountered. A discussion and amendment were performed, the scale was adapted and the improved version was used. Good psychometric properties have been reported for the BREAST-Q subscales in our pilot study (Cronbach's alpha was 0.89 to 0.92 , respectively, for the preoperative and postoperative modules).

Secondary sociodemographic, clinical and other psychosocial variables will be collected for all patients (table 2).

\section{Source of data}

Clinical data will be obtained by computerised clinical records (CCR), hosted on HP Doctor and Clinical Estation programmes that are being used in routine medical practice; sociodemographic, psychosocial and other health outcomes will be self-reported by the patient.

Collected individual patient data will be entered on paper case report forms and transferred to an Excel database. Data will be recorded in an anonymised format, using a unique alphanumeric study identification number on a secure database. Advanced data logic will be used such that only data fields relevant to the procedure and the indication selected will be displayed in later data collection forms. Participating researchers will also be required to maintain and securely store an Excel spreadsheet linking study ID numbers with the CCR patient number to allow long-term oncological outcomes to be evaluated at follow-up. Finally, the database will be migrated to SPSS V.22.00.

\section{Sample size}

Sample size calculations for the main aim of the study are based on findings from Scott $e t a \tilde{l}^{\tilde{7}}$ about HRQoL QLQ-C30 reference values. Assuming we would like to detect a difference of 15 points when comparing the iBR group and the dBR group and a difference of 10 points when comparing the iBR group and the 2sBR group, assuming a two-sided test with alpha $=0.05$, beta $=0.02$ (power $=0.08$ ) and expecting a $5 \%$ loss rate, then 38 and
86 patients per group will be necessary for the first and second comparison, respectively. Therefore, the sample size required will be 210 patients. The sample size required to perform the other objectives of the study is lower; thus, the estimated sample size enables us to address all the above objectives.

Patients will be recruited consecutively until the sample size is sufficient for each group, with a non-probabilistic sampling of the patients attended at the Breast Pathology Unit of La Paz University Hospital's Department of Gynecology. Considering our estimate, in 2016, 300 women were diagnosed with breast cancer at our hospital, $50 \%$ of whom underwent a mastectomy and $50 \%$ of whom are undergoing a BR. Therefore, the estimated sample size is appropriate to the recruitment needs from our centre. Sample size is calculated using Epi Info software. ${ }^{58}$

\section{Loss to follow-up}

A low 'lost to follow-up rate' will be essential in this type of study. The total loss to follow-up at the end of the study should be kept at less than $10 \%$ of the recruited population. However, we consider that this type of study performed under clinical conditions will include a low loss to follow-up.

\section{Statistical analysis}

An analysis of differences in characteristics between responders and non-responders will be performed with Student's t-test for normally distributed variables and the $\chi^{2}$ test for categorical variables, including a description of the profile of patients who abandon the study plus their reason for withdrawal.

All outcomes will be summarised using a descriptive analysis of each variable, overall and split by group (iBR, dBR and 2sBR). Normally distributed continuous outcomes will be summarised by the mean, $\mathrm{SD}$, minimum and maximum, and median and IQR for skewed data, and the qualitative variables will be expressed as frequencies and percentages. For comparing possible differences between groups at baseline, a one-way analysis of variance (ANOVA) and Tukey's post hoc analysis will be used for quantitative variables, and the Kruskal-Wallis test will be used for qualitative variables; the $\chi^{2}$ test will be used for categorical variables, according to data distribution.

The main aim of the study will be approached as follows:

In the first approach, the ANOVA and Tukey's post hoc analysis for quantitative variables or the Kruskal-Wallis test for qualitative variables will be used, and $\chi^{2}$ test will be used for categorical variables, according to data distribution. In the second approach, analysis of variance for repeated measures will be employed to test for differences between groups over time.

To analyse the sociodemographic, clinical and psychosocial factors associated with HRQoL, overall satisfaction with surgery and psychological impact, multivariable regression will be used to adjust for prognostic factors. To control for confounding effects, the model will be adjusted by age and by variables significantly related in the 


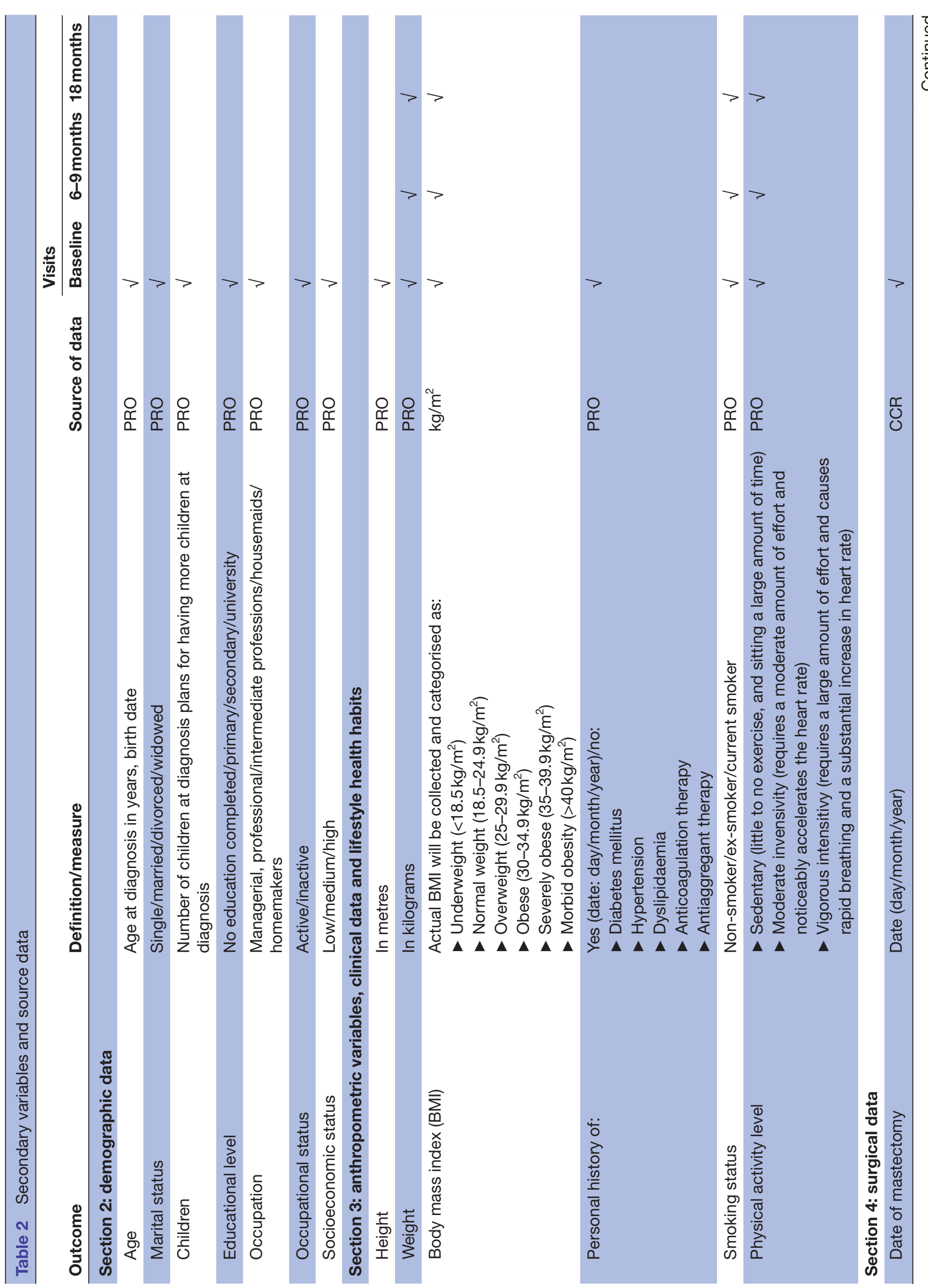



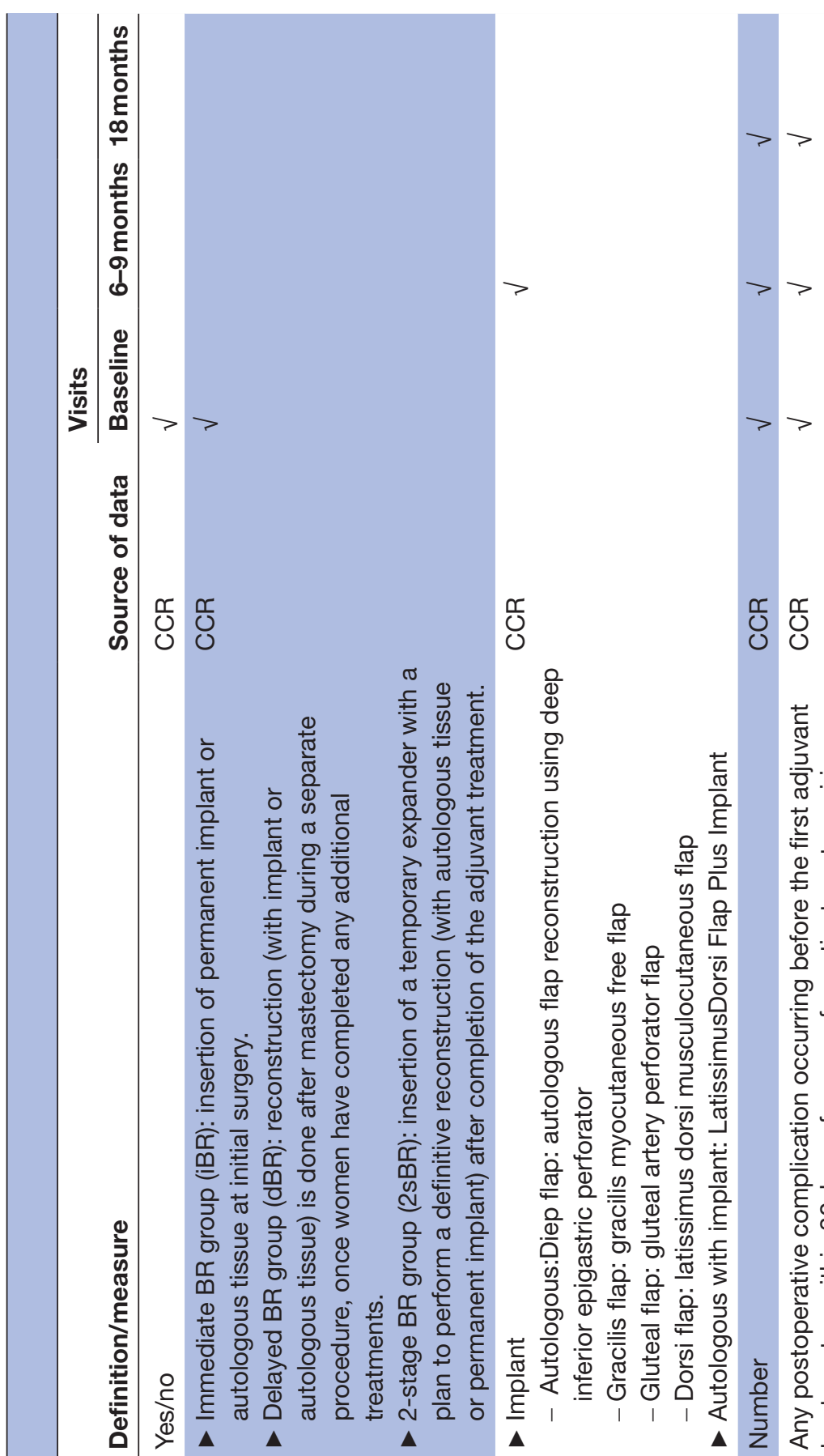


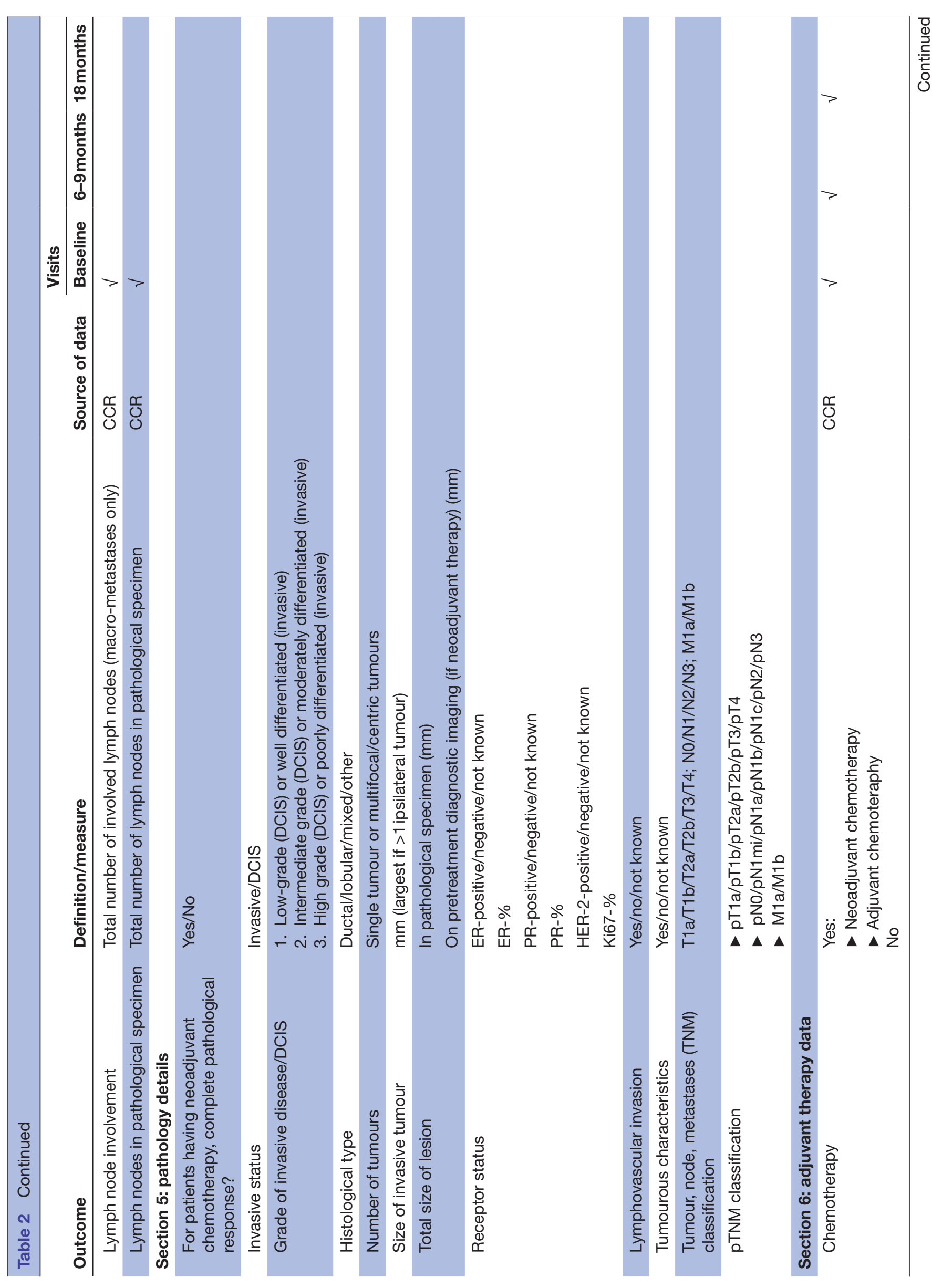

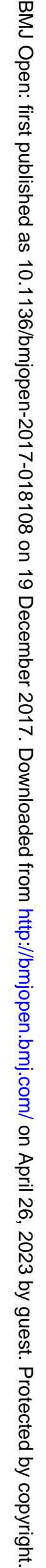




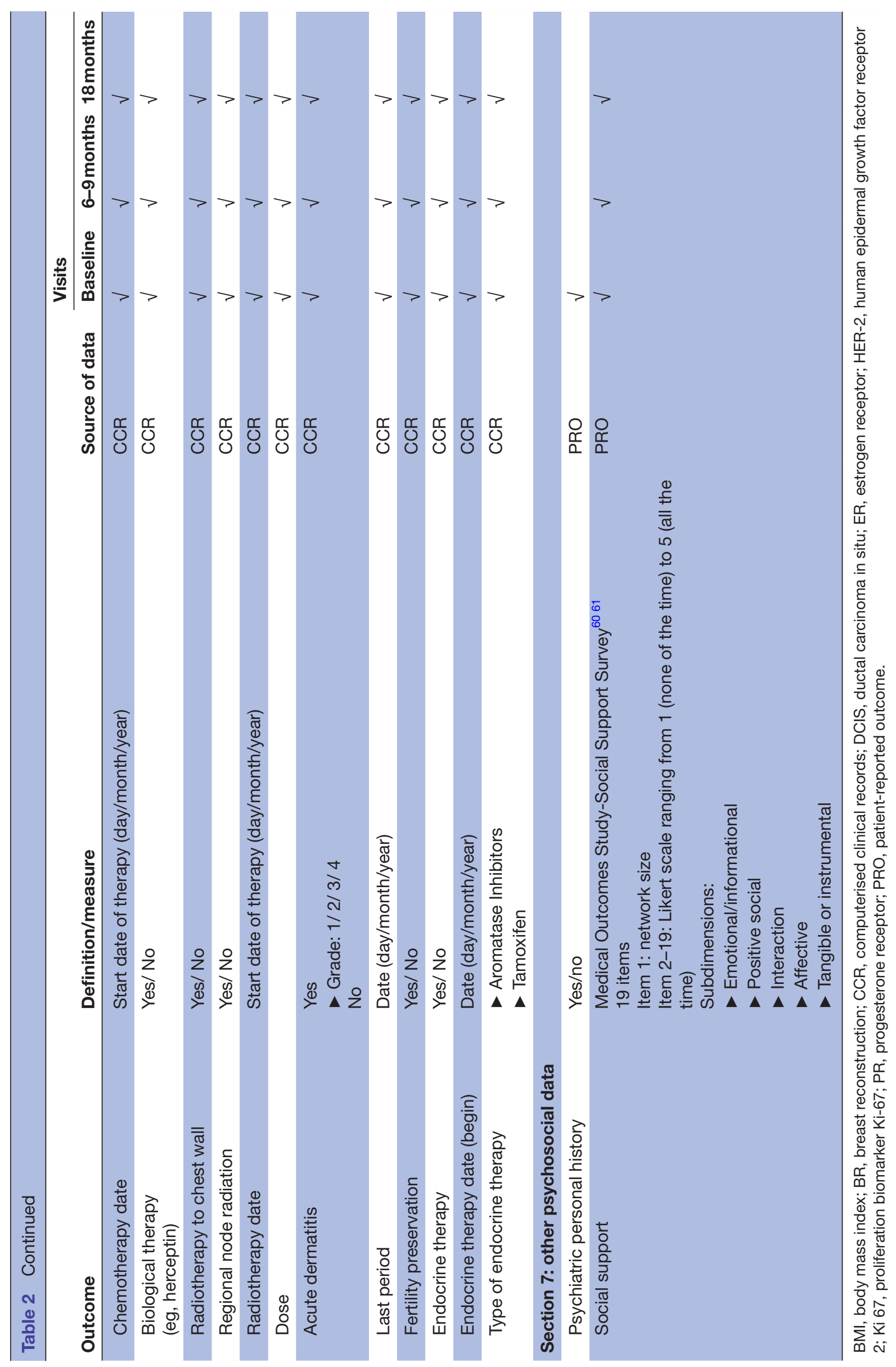

$\stackrel{\text { m }}{\longrightarrow}$

융

$\overrightarrow{\bar{F}}$

믐

तิ

ก)

$\vec{\circ}$

$\vec{\omega}$

윽 
bivariate analysis. Relevant variables from other studies already reported in the literature will also be taken into account. ${ }^{54}$

All analyses will be calculated with their $95 \%$ CI; statistical significance will be set at $\mathrm{P}<0.05$. Statistical processing of the data will be performed with SPSS software, version 22.00.61. ${ }^{59}$

\section{DISCUSSION}

This present study will address an important gap in the literature by answering a fundamental question regarding the patient-reported outcome of BR at a follow-up of 18 months. Currently, BR is considered a treatment and as a preventive measure for the potential psychological and physical damage associated with mastectomy in women with breast cancer. This procedure is not harmless, however, and previous research suggests discordant results.

Reaching a consensus as to the optimal time for the BR (immediate, deferred or in two stages) is one of the main objectives in this field. Multiple variables are involved: surgical equipment preference, type of tissue used for reconstruction (autologous, alloplastic or mixed), medical factors and patient's preference; the need for radiotherapy is the fundamental aspect on which many medical teams base their decision about when and how BR should be performed. There is general consensus around the fact that radiotherapy increases the complications associated with BR; however, there are contradictory results as to whether the timing of reconstruction modifies the likelihood of such complications.

The present study is strengthened by its follow-up nature, allowing us to draw conclusions about causality. However, some limitations must be acknowledged. The study will be performed based on a clinical cohort of patients with breast cancer at La Paz University Hospital. Because it is a sample of convenience, not population based, it might not be representative of the entire population. However, our hospital is a reference centre for this type of disorder in the Madrid region, and our population could be representative of this group of patients. In addition, although both the HRQoL and psychosocial adjustment scales are well validated in Spanish populations, the BREAST-Q has not undergone a formal validation in a Spanish population; therefore, its validity might be negatively affected in the present population. The Spanish version of the BRECAR, however, has been forward and backward translated for the present study. The period between the baseline visit and the date of mastectomy would be different for each patient, considering, for example, time on the waiting list for the surgical procedure; however, this time would not exceed 3 or 4 weeks for all the patients, so the variability would be minimal. The classification of the patients in the groups according to BR timing will be performed under routine medical conditions. A clinical trial is not possible in this type of study, however, because the surgical procedure cannot be randomised. Therefore, subgroup analysis will be performed comparing baseline characteristics and will be adjusted in the multivariable analysis considering those variables with significant differences.

This prospective study, with short-term and medium-term follow-ups, will aid the creation of a tool to help in clinical decision making and to determine the timing of maximum psychological vulnerability during the various stages of reconstruction, allowing the establishment of specific care plans for women with breast cancer.

Due to possible limitations, and taking into account the gap in scientific literature on prospective studies focusing on how the decision of the type and timing of BR affect the patient's HRQoL, self-esteem, body image and satisfaction with the surgery, the BRECAR study is presented as the first prospective study on BR performed in our country. Considering various clinical aspects, this study combines quality of life, satisfaction and psychosocial adjustment of the patient as variables that determine the clinical success of $B R$ and analyses the influence of the type and timing of reconstruction. In order to assess the patient's satisfaction about surgery, we will use the BREAST-Q survey, which has been validated for the Spanish language for the first time.

\section{Ethics and dissemination}

All patients will provide an informed consent in accordance with the hospital's ethics guidelines. Research protocols will follow ethical standards as outlined in the Declaration of Helsinki. Procedure within this research project will be conducted in accordance with the guidelines for Good Clinical Practice.

The protocol will be disseminated via journal articles and conference presentations. Collective data will be analysed, and the results of the study presented at appropriate scientific meetings and published in peer-reviewed journals. The results can then be used to inform patients and surgeons and aid decision making for women considering BR.

Acknowledgements We are further grateful to the BRECAR Study Group members of La Paz University Hospital (Madrid, Spain): C Casado Sánchez, L Landín Jarillo, S Zarbasch Etemadi, L Miralles Olivar, A Loayza Galindo, J I Sánchez Méndez, C Martí Álvarez, M García Redondo, E York Pineda, A Ballesteros Gil and M A Rodríguez Patón.

Contributors MHdIM and PG-C conceived the study and performed its design. All authors drafted the manuscript. All authors read and approved the final manuscript.

Funding The study is funded by La Paz University Hospital's Institute for Health Research (IdiPAZ) under the Established Group Grant 2015. The financial contributors did not have any involvement in the study.

Competing interests None declared.

Patient consent Obtained.

Ethics approval The study protocol has been approved by the institutional review board of La Paz Hospital (no. Pl-2036).

Provenance and peer review Not commissioned; externally peer reviewed.

Open Access This is an Open Access article distributed in accordance with the Creative Commons Attribution Non Commercial (CC BY-NC 4.0) license, which permits others to distribute, remix, adapt, build upon this work non-commercially, and license their derivative works on different terms, provided the original work is properly cited and the use is non-commercial. See: http://creativecommons.org/ licenses/by-nc/4.0/ 
(c) Article author(s) (or their employer(s) unless otherwise stated in the text of the article) 2017. All rights reserved. No commercial use is permitted unless otherwise expressly granted.

\section{REFERENCES}

1. Ferlay J, Soerjomataram I, Ervik M, et al. Incidence and mortality and epidemiology of breast cancer in the world. At Cancer Incidence and Mortality Worldwide, 2012. http://globocan.iarc.fr.

2. INE. Boletín informativo del Instituto Nacional de Estadística 7/12: Instituto Nacional de Estadística. http://www.ine.es

3. Howard MA, Sisco M, Yao K, et al. Patient satisfaction with nipplesparing mastectomy: A prospective study of patient reported outcomes using the BREAST-Q. J Surg Oncol 2016;114:416-22.

4. AECEP. Sociedad Española de Cirugía Plástica Reparadora y Estética. 2017 http://www. http://aecep.es

5. Nguyen KT, Hanwright PJ, Smetona JT, et al. Body mass index as a continuous predictor of outcomes after expander-implant breast reconstruction. Ann Plast Surg 2014;73:19-24.

6. Lundberg J, Thorarinsson A, Karlsson P, et al. When is the deep inferior epigastric artery flap indicated for breast reconstruction in patients not treated with radiotherapy? Ann Plast Surg 2014;73:105-13.

7. Rao S, Stolle EC, Sher S, et al. A multiple logistic regression analysis of complications following microsurgical breast reconstruction. Gland Surg 2014;3:226-3.

8. Antoniuk PM. Breast reconstruction. Obstet Gynecol Clin North Am 2002;29:209-23.

9. Petit J, Rietjens M, Garusi C. Breast reconstructive techniques in cancer patients: which ones, when to apply, which immediate and long term risks? Crit Rev Oncol Hematol 2001;38:231-9.

10. Juhl AA, Christensen S, Zachariae R, et al. Unilateral breast reconstruction after mastectomy - patient satisfaction, aesthetic outcome and quality of life. Acta Oncol 2017;56:225-31.

11. Giménez-Climent MJ, et al. Reunión de consenso sobre la reconstrucción postmastectomía. Revista de Senología y Patología Mamaria 2008;21:106-12.

12. Reza Goyanes M, Andradas Aragonés E, Blasco Amaro JA, et al. Revisión sistemática y evaluación de resultados en una unidad de RMI de la Comunidad de Madrid. Unidad de Evaluación de Tecnologías Sanitarias (UETS: Agencia Laín Entralgo, 2005.

13. Petit J, Rietjens M, Garusi C. Breast reconstructive techniques in cancer patients: which ones, when to apply, which immediate and long term risks? Crit Rev Oncol Hematol 2001;38:231-9.

14. Goldhirsch A, Winer EP, Coates AS, et al. Personalizing the treatment of women with early breast cancer: highlights of the St Gallen International Expert Consensus on the Primary Therapy of Early Breast Cancer 2013. Ann Oncol 2013;24:2206-23.

15. Chuba PJ, Stefani WA, Dul C, et al. Radiation and depression associated with complications of tissue expander reconstruction. Breast Cancer Res Treat 2017;164:641-7.

16. Barry M, Kell MR. Radiotherapy and breast reconstruction: a metaanalysis. Breast Cancer Res Treat 2011;127:15-22.

17. Schaverien MV, Macmillan RD, McCulley SJ. Is immediate autologous breast reconstruction with postoperative radiotherapy good practice?: a systematic review of the literature. J Plast Reconstr Aesthet Surg 2013;66:1637-51.

18. Mirzabeigi MN, Smartt JM, Nelson JA, et al. An assessment of the risks and benefits of immediate autologous breast reconstruction in patients undergoing postmastectomy radiation therapy. Ann Plast Surg 2013;71:149-55.

19. Clarke-Pearson EM, Chadha M, Dayan E, et al. Comparison of irradiated versus nonirradiated DIEP flaps in patients undergoing immediate bilateral DIEP reconstruction with unilateral postmastectomy radiation therapy (PMRT). Ann Plast Surg 2013;71:250-4.

20. Berbers J, van Baardwijk A, Houben R, et al. 'Reconstruction: before or after postmastectomy radiotherapy?' A systematic review of the literature. Eur J Cancer 2014;50:2752-62.

21. Yan C, Fischer JP, Freedman GM, et al. The timing of breast irradiation in two-stage expander/implant breast reconstruction. Breast J 2016;22:322-9.

22. Lipa JE, Qiu W, Huang N, et al. Pathogenesis of radiation-induced capsular contracture in tissue expander and implant breast reconstruction. Plast Reconstr Surg 2010;125:437-45.

23. Collier P, Williams J, Edhayan G, et al. The effect of timing of postmastectomy radiation on implant-based breast reconstruction: a retrospective comparison of complication outcomes. Am J Surg 2014;207:408-11.
24. Razdan SN, Cordeiro PG, Albornoz CR, et al. Cost-effectiveness analysis of breast reconstruction options in the setting of postmastectomy radiotherapy using the BREAST-Q. Plast Reconstr Surg 2015;136:88-9.

25. Ho AL, Bovill ES, Macadam SA, et al. Postmastectomy radiation therapy after immediate two-stage tissue expander/implant breast reconstruction. Plast Reconstr Surg 2014;134:1e-10.

26. Yan C, Fischer JP, Wes AM, et al. The cost of major complications associated with immediate two-stage expander/implant-based breast reconstruction. J Plast Surg Hand Surg 2015;49:166-71.

27. Mesbahi AN, McCarthy CM, Disa JJ. Breast reconstruction with prosthetic implants. Cancer J 2008;14:230-5.

28. Berterö C, Chamberlain Wilmoth M. Breast cancer diagnosis and its treatment affecting the self: a meta-synthesis. Cancer Nurs 2007;30:194-202.

29. Wilmoth MC. The aftermath of breast cancer: an altered sexual self Cancer Nurs 2001;24:278-86.

30. Fobair P, Stewart SL, Chang S, et al. Body image and sexual problems in young women with breast cancer. Psychooncology 2006;15:579-94.

31. Ganz PA, Rowland JH, Desmond K, et al. Life after breast cancer: understanding women's health-related quality of life and sexual functioning. J Clin Oncol 1998;16:501-14.

32. Liu C, Zhuang Y, Momeni A, et al. Quality of life and patient satisfaction after microsurgical abdominal flap versus staged expander/implant breast reconstruction: a critical study of unilateral immediate breast reconstruction using patient-reported outcomes instrument BREAST-Q. Breast Cancer Res Treat 2014;146:117-26.

33. Nano MT, Gill PG, Kollias J, et al. Psychological impact and cosmetic outcome of surgical breast cancer strategies. ANZ J Surg 2005;75:940-7.

34. Dauplat J, Kwiatkowski F, Rouanet P, et al. Quality of life after mastectomy with or without immediate breast reconstruction. $\mathrm{Br} \mathrm{J}$ Surg 2017;104:1197-206.

35. de Raaff CA, Derks EA, Torensma B, et al. Breast reconstruction afte mastectomy: does it decrease depression at the long-term? Gland Surg 2016;5:377-84.

36. Flitcroft K, Brennan M, Spillane A. Making decisions about breast reconstruction: a systematic review of patient-reported factors influencing choice. Quality of Life Research 2017;26:2287-319.

37. Fernández-Delgado J, López-Pedraza MJ, Blasco JA, et al. Satisfaction with and psychological impact of immediate and deferred breast reconstruction. Ann Oncol 2008;19:1430-4.

38. Schain WS, Wellisch DK, Pasnau RO, et al. The sooner the better: a study of psychological factors in women undergoing immediate versus delayed breast reconstruction. Am J Psychiatry 1985:142:40-6.

39. Rosson GD, Shridharani SM, Magarakis M, et al. Quality of life before reconstructive breast surgery: a preoperative comparison of patients with immediate, delayed, and major revision reconstruction. Microsurgery 2013;33:253-8.

40. Metcalfe KA, Semple J, Quan ML, et al. Changes in psychosocial functioning 1 year after mastectomy alone, delayed breast reconstruction, or immediate breast reconstruction. Ann Surg Oncol 2012;19:233-41.

41. Teo I, Reece GP, Christie IC, et al. Body image and quality of life of breast cancer patients: influence of timing and stage of breast reconstruction. Psychooncology 2016;25:1106-12.

42. Brandberg $Y$, Malm M, Blomqvist L. A prospective and randomized study, "SVEA," comparing effects of three methods for delayed breast reconstruction on quality of life, patient-defined problem areas of life, and cosmetic result. Plast Reconstr Surg 2000;105:66-74.

43. Elder EE, Brandberg Y, Björklund T, et al. Quality of life and patient satisfaction in breast cancer patients after immediate breast reconstruction: a prospective study. Breast 2005;14:201-8.

44. Gopie JP, ter Kuile MM, Timman R, et al. Impact of delayed implant and DIEP flap breast reconstruction on body image and sexual satisfaction: a prospective follow-up study. Psychooncology 2014;23:100-7.

45. Aaronson NK, Ahmedzai S, Bergman B, et al. The European Organization for Research and Treatment of Cancer QLQ-C30: a quality-of-life instrument for use in international clinical trials in oncology. J Natl Cancer Inst 1993;85:365-76.

46. EORTC Quality of life. EORTC QLQ-C30. http://groups.eortc.be/qol/ eortc-qlq-c30

47. Arraras Jl. Cuestionario de Calidad de Vida de la EORTC para cáncer de mama EORTC QLQ-C23. Psicología Conductual 2001;9:81-98.

48. Sprangers MA, Groenvold M, Arraras JI, et al. The European Organization for Research and Treatment of Cancer breast cancerspecific quality-of-life questionnaire module: first results from a three-country field study. J Clin Oncol 1996;14:2756-68. 
49. Hopwood P, Fletcher I, Lee A, et al. A body image scale for use with cancer patients. Eur J Cancer 2001;37:189-97.

50. Gómez-Campelo P, Bragado-Álvarez C, Hernández-Lloreda MJ, et al. The Spanish version of the Body Image Scale (S-BIS): psychometric properties in a sample of breast and gynaecological cancer patients. Support Care Cancer 2015;23:473-81.

51. Echeburúa E. Evaluación y tratamiento de la Fobia social. Barcelona: Biblioteca de Psicología, Psiquiatría y Salud, 1995.

52. Rosenberg M. Society and the adolescence self-image. Princeton University Press: Princeston NJ, 1965.

53. Kroenke K, Spitzer RL, Williams JB. The PHQ-9: validity of a brief depression severity measure. J Gen Intern Med 2000; 16:606-13.

54. Diez-Quevedo C, Rangil T, Sanchez-Planell L, et al. Validation and utility of the patient health questionnaire in diagnosing mental disorders in 1003 general hospital Spanish inpatients. Psychosom Med 2001;63:679-86.
55. Pusic AL, Cano S, Klassen A. BREAST-Q. https://eprovide.mapitrust.org/instruments/breast-q.

56. Acquadro C, Conway K, Giroudet C, et al. Linguist ic validation Manual for Health Outcome Assessment. Mapi Institute, 2012.

57. Scott NW, Fayers PM, Aaronson NK, et al. EORTC QLQ-C30

Reference Values. http://groups.eortc.be/qol/sites/default/files/img/ newsletter/reference_values_manual2008.pdf.

58. CDC-INFO. Epi Info. 2017 https://www.cdc.gov/epiinfo/index.html.

59. IBM Corp. Released 2013. IBM SPSS Statistics for Windows, Version 22.0. Armonk, NY: IBM Corp.

60. Sherbourne CD, Stewart AL. The MOS social support survey. Soc Sci Med 1991;32:705-14.

61. de la Revilla L, Luna J, Baillón E, et al. Validación del cuestionario MOS de apoyo social en Atención Primaria. Medicina Familia 2005;6:10-18. 